\section{OPEN ACCESS}

Edited by:

Maoli Duan,

Karolinska Institutet (KI), Sweden

Reviewed by:

Bryan Kevin Ward, Johns Hopkins University,

United States

Jeremy Hornibrook,

University of Canterbury, New Zealand

${ }^{*}$ Correspondence:

Zhengnong Chen

jassey@126.com

Dongzhen Yu

drdzyu@126.com

†These authors have contributed equally to this work

Specialty section:

This article was submitted to

Neuro-Otology,

a section of the journal

Frontiers in Neurology

Received: 19 October 2021

Accepted: 13 January 2022

Published: 04 February 2022

Citation:

Jiang Y, Xu M, Yao Q, Li Z, Wu Y, Chen Z, Yu D, Shi H and Yin S (2022) Changes of Vestibular Symptoms in Menière's Disease After Triple

Semicircular Canal Occlusion: A

Long-Term Follow-Up Study.

Front. Neurol. 13:797699.

doi: 10.3389/fneur.2022.797699

\title{
Changes of Vestibular Symptoms in Menière's Disease After Triple Semicircular Canal Occlusion: A Long-Term Follow-Up Study
}

Yumeng Jiang ${ }^{1,2,3 \dagger}$, Maoxiang $\mathrm{X} \mathrm{u}^{1,2,3 \dagger}$, Qingxiu Yao ${ }^{1,2,3 \dagger}$, Zhuangzhuang $\mathrm{Li}^{1,2,3}$, Yaqin $\mathrm{Wu}^{1,2,3}$, Zhengnong Chen ${ }^{1,2,3 *}$, Dongzhen Yu ${ }^{1,2,3 *}$, Haibo Shi ${ }^{1,2,3}$ and Shankai Yin ${ }^{1,2,3}$

\footnotetext{
'Department of Otolaryngology-Head and Neck Surgery, Shanghai Jiao Tong University Affiliated Sixth People's Hospital, Shanghai, China, ${ }^{2}$ Otolaryngology Institute of Shanghai Jiao Tong University, Shanghai, China, ${ }^{3}$ Shanghai Key Laboratory of Sleep Disordered Breathing, Shanghai, China
}

Background: The clinical efficacy of triple semicircular canal occlusion (TSCO) and vestibular nerve resection (VNS) for patients with Ménière's disease has been unclear.

Objective: To explore changes in vestibular symptoms after TSCO and its advantages compared to the classical operation of VNS in patients with Menière's disease.

Methods: In total, 36 patients with Menière's disease performed TSCO or VNS at Shanghai Jiao Tong University Affiliated Sixth People's Hospital, China from May 2005 to July 2021, and all of them were enrolled in our study. Twelve of them underwent TSCO, 23 underwent VNS, and 1 had both treatments. We compared the demographic parameters, clinical symptoms, and selected test results between the two surgical methods. Ten patients each who underwent TSCO and VNS completed the follow-up. We collected and compared data pertaining to changes in vestibular symptoms.

Results: No significant difference in demographic parameters, clinical symptoms, or auditory or vestibular test results was detected between the two groups preoperatively. The TSCO group with vertigo as the main complaint experienced less residual paroxysmal dizziness after surgery than the VNS group $(P=0.020)$. Also, $57 \%$ of the patients in the VNS group had unsteadiness after surgery, while no such problems were reported in the TSCO group $(P=0.025)$.

Conclusions: Our study shows that TSCO controls vertigo in most Menière's disease patients, and also has the advantage of lower rates of postoperative paroxysmal dizziness and unsteadiness than VNS. Thus, TSCO may be an effective surgery for refractory Menière's disease.

Keywords: vestibular symptoms, triple semicircular canal occlusion, vestibular nerve section, Menière's disease, clinical benefit 


\section{INTRODUCTION}

Menière's disease is an inner ear disorder characterized by two or more episodes of vertigo associated with fluctuating lowand medium-frequency hearing loss (HL), tinnitus, and aural fullness. The duration of vertigo attacks is between $20 \mathrm{~min}$ and $12 \mathrm{~h}$ (1). An epidemiological survey in Japan showed that 50-200 out of 100,000 adults suffer from Menière's disease, which mostly occurs in people aged 40-60 years $(2,3)$. The disease is also characterized by an impaired quality of life (4) and can lead to restricted activity due to refractory vertigo (5).

Both medical and surgical interventions are used in the treatment of Menière's disease, to control vertigo attacks and preserve hearing and vestibular function (6). A series of relatively reliable treatments has been developed for Menière's disease (3). Improving lifestyle and exercising more is the initial treatment step. Previous studies reported that a low-salt diet free from caffeine can help balance the ion concentration in the endolymph of the inner ear (7). The next step of treatment is to reduce the number of attacks and relieve vertigo and dizziness symptoms via medications including betahistine, antihistamines, benzodiazepines, ginkgo biloba extract, and corticosteroids (8); diuretics may also be tried to reduce endolymphatic hydrops (9). Most of the symptoms can be stably controlled by medicine, although $15-40 \%$ of patients suffer disabling refractory vertigo (7).

For those living with serious vertigo attacks for years, and in whom lifestyle changes and medical therapy have been tried unsuccessfully various surgical treatments are available according to the patient's hearing status (3). Endolymphatic sac surgery (ESS), labyrinthectomy, and vestibular nerve resection (VNS) were recommended by the AAO-HNS clinical guidelines as early as 1985 (10). Dandy performed the first vestibular nerve section in 1942 and advocated selective nerve section for intractable Menière's disease thereafter (11). In 1990 Parnes and McClure described posterior semicircular canal occlusion for benign paroxysmal positional vertigo (12). The purpose is to eliminate the movement endolymph in the canals, so as to eliminate the vertigo attack caused by specific head movement in patients with BPPV. We hypothesize the pathophysiology of Menière's disease is that excessive accumulation of endolymph can cause bulk endolymph movement and episodes of spinning vertigo, and this can be improved by semicircular canal obstruction. Triple semicircular canal occlusion (TSCO) for Menière's disease was advocated by Yin et al. (13), and has been performed in China and elsewhere with good vertigo control and preservation of hearing $(14,15)$.

However, due to the lack of comparison of clinical efficacy between different surgical methods, it is difficult to determine the optimal approach for refractory Menière's disease (16). Therefore, the aim of the present study is to evaluate the effectiveness of TSCO to control vertigo attacks over the long term and improve quality of life compared to traditional VNS.

\section{METHODS}

\section{Study Design}

A retrospective study was performed of patients with Menière's disease in Shanghai Jiao Tong University Affiliated Sixth People's Hospital, China from May 2005 to July 2021. The indications for surgery were a pure-tone average of $>70 \mathrm{~dB}$ Hearing Level (HL) (according to the AAO-HNS clinical guidelines), failure of previous medical therapy applied over at least 6 months, and a strong desire for surgery. The exclusion criteria included a history of any other neurogenic disease, vestibular neuritis, brain tumor, vestibular migraine, BPPV, or any other disease that can cause vertigo.

Before surgery, all patients underwent a series of auditory and vestibular evaluations, including pure-tone audiometry, tympanometry, distortion product otoacoustic emission and glycerol test. Tympanometry is used to test the condition of the middle ear by creating air pressure in the ear canal. Distortion product otoacoustic emission, otoacoustic emission evoked by two pure tones, reflect the integrity of outer hair cell and the function of cochlea (17). The glycerol test was regarded as positive in the audiometry if the pure tone threshold improved at least $15 \mathrm{~dB}$ at minimum 3 frequencies after ingesting glycerol (18). Patients seen within the last 3 years also underwent radiological examination, including temporal bone computed tomography and magnetic resonance imaging, and intratympanic gadolinium-enhanced inner-ear magnetic resonance imaging.

Changes of vestibular symptoms were evaluated by telephone follow-up, which inquired about attacks of vertigo after surgery and other symptoms. The specific questions are as follows: Would you please describe the change of your vestibular symptoms during these year after surgery; Was there any vertigo attacked again after surgery and when? Were there any symptoms such as dizziness and unsteadiness appeared? When and how it happened; How about your hearing condition? We calculated the average number of attacks for each ear. In July 2021, we administered four questionnaires by telephone to each TSCO patient: the Dizziness Handicap Inventory (DHI), ActivitiesSpecific Balance Confidence Scale (ABC), Tinnitus Handicap Inventory (THI), and Visual Analog Scale (VAS).

The study was designed and executed in accordance with the recommendations of the Ethics Committee of Shanghai Jiao Tong University Affiliated Sixth People's Hospital (2020 KY 004).

\section{Patients and Outcome Measures}

In the past 16 years, 99 Menière's disease patients underwent surgery, including TSCO, VNS, and ESS, at Shanghai Jiao Tong University Affiliated Sixth People's Hospital. Of these 99 patients, 36 patients underwent TSCO or VNS and all were included in our retrospective study. Ten patients per surgical method who completed follow-up were included and analyzed in this study in terms of the curative effect of surgery, The remaining 16 patients were unable to be contacted or had not returned to our hospital.

Vestibular symptoms, including vertigo, dizziness, and unsteadiness, were assessed before and after surgery (19). The primary outcome was vertigo control as determined by 
the number of vertigo episodes. According to the American Academy of Otolaryngology-Head and Neck Surgery guidelines (1), the patient's vertigo control status was determined by comparison of the frequency of vertigo attacks between 6 months before and 18-24 months after surgery. Vertigo control was expressed numerically (number of attacks after treatment divided by the number prior to the treatment) as follows; $0=\mathrm{A}$, complete control; $1-41=\mathrm{B}$, substantial control; $41-80=\mathrm{C}$, limited control; 81-120 = D, insignificant control; and $>120$ $=\mathrm{E}$, no control. The postoperative data of all patients were collected by telephone in July 2021. Dizziness and unsteadiness were secondary outcomes. In the TSCO group, the DHI, ABC, and VAS scores were used to analyze secondary outcomes by evaluating vertigo, dizziness and balance; the THI score was used to evaluate the severity of tinnitus. The DHI, a questionnaire exploring the self-perceived handicapping effects of vestibular disease consists of three parts (DHI-P, -E and -F) evaluated separately (20). Visual analog scale (VAS) was administered via telephone to measure symptoms or conditions that are not captured by the other measures. We asked the patient to rate their current symptom level on a scale from 0 to 10 , with 10 being the patient's symptom at the time when he was most affected by Menière's disease before surgery, and 0 being the patient's symptom when completely healthy. For the VNS group, clinical information including vertigo attacks, unsteadiness, HL, and tinnitus was collected by telephone or outpatient follow-up. All of the information was collected in July 2021.

Hearing (clinical stage) was assessed according to the puretone average at frequencies of $0.5,1$, and $2 \mathrm{kHz}$; this also informed the surgical method, along with clinical guidelines. The hearing of patients was categorized as follows: $0-25 \mathrm{~dB} \mathrm{HL}=$ stage $1 ; 26-$ $40 \mathrm{~dB} \mathrm{HL}=$ stage $2 ; 41-70 \mathrm{~dB} \mathrm{HL}=$ stage 3 ; and $>70 \mathrm{~dB} \mathrm{HL}=$ stage 4 .

\section{Surgical Protocol for TSCO}

Yin et al. $(13,14,21)$ were the first to modify SCO, which was originally performed in patients with BPPV, and apply it to Menière's disease in 2004. After standard mastoidectomy, three semicircular canals are contoured and a bone island with a diameter of approximately $1 \mathrm{~mm}$ is drilled in the non-ampullary arm of each canal. Then, great care is taken while prying off each bone island with a hook, and each canal is completely occluded with a piece of muscle or fascia tissue (Figure 1A). Next, the access area is covered by a piece of fascia with some bone dust sprayed on the muscle tissue. Finally, a piece of gelatin sponge is placed on the surgical site.

\section{Surgical Protocol for VNS}

Retrolabyrinthine and retrosigmoid approaches were used in the VNS group (22). The difference between these approaches lies in the location in which the dura mater is exposed. The first approach contours the mastoid and opens the internal auditory canal to $270^{\circ}$. The second approach opens a bone window approximately $3 \mathrm{~cm} \times 4 \mathrm{~cm}$ in size at the junction of the parietal, occipital, and temporal bones. Then, the dura mater is opened, and cerebrospinal fluid released. The cerebellum is gently pressed to expose the cerebellopontine angle and separate the vestibular and cochlear nerves. The vestibular nerve is then cut. After cleaning the cavity the surgical site is filled with hemostatic silk and gelatin sponge (23) (Figure 1B).

\section{Statistical Analysis}

Statistical analysis was performed with SPSS software (version 25.0; IBM Corp., Armonk, NY, USA). The normality of continuous variables was assessed with the Shapiro-Wilk test. We express normally distributed variables as mean \pm standard deviation, and compared the two groups using independent sample $t$-tests; we express non-normally distributed variables as mean \pm standard deviation, and used nonparametric tests (Mann-Whitney $\mathrm{U}$ test) to compare the groups. We express categorical variables as frequency or percentage and used the chi-square test to compare the groups. We considered $P<0.05$ statistically significant.

\section{RESULTS}

\section{Clinical Symptoms and Tests}

During the past 16 years, 99 Menière's disease patients underwent surgery in Shanghai Jiao Tong University Affiliated Sixth People's Hospital, of which 12 and 23 underwent TSCO and VNS, respectively (one patient underwent VNS in 2005 and TSCO in 2017), and were included in our retrospective study. There were no statistically significant differences between the two groups in demographic parameters, including gender and age at surgery, or clinical symptoms, including duration of vertigo attack, number of years of attacks, number of vertigo spells in the 6 months before surgery, worst hearing level, number of years of HL, tinnitus, or affected side (all $P>0.05$; Table 1). Similarly, no significant differences between the groups were detected in the results of auditory tests before surgery, including tympanometry, distortion product otoacoustic emission and glycerol tests.

\section{Vertigo Control}

According to the American Academy of Otolaryngology-Head and Neck Surgery guidelines (1), patients who underwent surgery more than 24 months ago $(N=4$ and 10 in the TSCO and VNS groups, respectively) were analyzed in terms of post-operation vertigo within 2 years; $100 \%$ of the patients in the TSCO group achieved complete control of vertigo, compared to $90 \%$ in the VNS group (10\% achieved substantial control). During the entire follow-up period, four patients in the TSCO group and seven in the VNS group had no vertigo attacks, and three patients in the VNS group had a vertigo attack. There was no significant difference in number of postoperative vertigo episodes in 24 months between the two surgical methods using independent sample $t$-tests $(P=0.839)$. In brief, the surgical methods had similar efficacy for vertigo attack control (number of attacks after treatment divided by the number prior to the treatment) for most patients in two group got an A control in 2 years after surgery using chi-square test $(P=0.512$; Table 2; Figure 2A). Six patients in the TSCO group underwent surgery $<24$ months before this study was completed, and $50 \%$ of them had no vertigo attacks after surgery. Although the other $50 \%$ of these patients had vertigo attacks, their frequency was much lower than before 

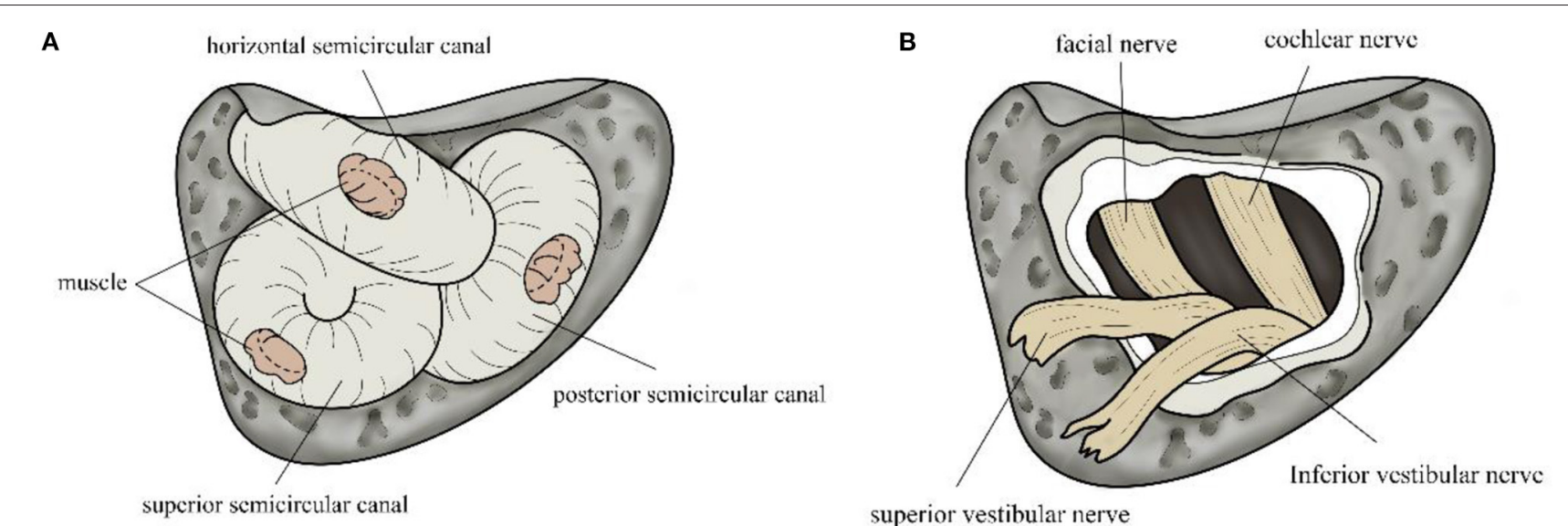

FIGURE 1 | Diagram of triple semicircular canal occlusion and vestibular nerve resection. (A) Muscle tissue blocks the three semicircular canals, which are clearly contoured. (B) The vestibular is cut off after being separated from the cochlear nerve.

TABLE 1 | Patient demographics, symptoms, and results of tests before surgery.

\begin{tabular}{|c|c|c|c|c|}
\hline & Total $\left(N=35^{\star}\right)$ & $\operatorname{TSCO}(N=12)$ & VNS $(N=23)$ & $P$-value \\
\hline \multicolumn{5}{|l|}{ Gender } \\
\hline Male (n/\%) & $14(40 \%)$ & $5(42 \%)$ & $9(39 \%)$ & 0.884 \\
\hline Female $(n / \%)$ & $21(60 \%)$ & $7(58 \%)$ & $14(61 \%)$ & \\
\hline Age at surgery (years) & $53.46 \pm 9.77$ & $53.58 \pm 10.92$ & $53.39 \pm 9.38$ & 0.957 \\
\hline \multicolumn{5}{|l|}{ Side } \\
\hline Right $(n / \%)$ & $17(49 \%)$ & $7(58 \%)$ & $10(43 \%)$ & 0.404 \\
\hline Left $(n / \%)$ & $18(51 \%)$ & $5(42 \%)$ & $13(57 \%)$ & \\
\hline \multicolumn{5}{|l|}{ Vertigo } \\
\hline Duration (years) & $7.55 \pm 7.12$ & $6.89 \pm 3.59$ & $7.91 \pm 8.52$ & 0.68 \\
\hline Average duration of vertigo episode(h) & $5.48 \pm 6.58$ & $2.78 \pm 3.13$ & $4.90 \pm 5.81$ & 0.65 \\
\hline Number of spells 6 months before surgery $(n)$ & $15.26 \pm 10.93$ & $16.25 \pm 11.68$ & $14.73 \pm 10.74$ & 0.71 \\
\hline \multicolumn{5}{|l|}{ Hearing loss } \\
\hline Worst hearing (dB) & $67.26 \pm 20.61$ & $69.58 \pm 13.37$ & $66.04 \pm 23.71$ & 0.637 \\
\hline Duration (years) & $6.75 \pm 6.92$ & $5.53 \pm 3.32$ & $7.48 \pm 8.38$ & 0.99 \\
\hline \multicolumn{5}{|l|}{ Tinnitus } \\
\hline Duration (years) & $8.42 \pm 10.27$ & $8.56 \pm 13.60$ & $8.34 \pm 8.29$ & 0.49 \\
\hline \multicolumn{5}{|l|}{ Test } \\
\hline \multicolumn{5}{|l|}{ Tympanogram } \\
\hline $\mathrm{A}(n / \%)$ & $21(91 \%)$ & $10(91 \%)$ & $11(92 \%)$ & 0.949 \\
\hline As $(n / \%)$ & $2(9 \%)$ & $1(9 \%)$ & $1(8 \%)$ & \\
\hline \multicolumn{5}{|l|}{ Distortion product otoacoustic emission $(n / \%)$} \\
\hline Present & $1(6 \%)$ & $0(0 \%)$ & $1(12.5 \%)$ & 0.274 \\
\hline Absent & 16(94\%) & $9(100 \%)$ & $7(87.5 \%)$ & \\
\hline \multicolumn{5}{|l|}{ Glycerol test $(n / \%)$} \\
\hline Positive & $10(77 \%)$ & $3(60 \%)$ & $7(87.5 \%)$ & 0.252 \\
\hline Negative & $3(23 \%)$ & $2(40 \%)$ & $1(12.5 \%)$ & \\
\hline
\end{tabular}

*One patient who underwent both triple semicircular canal occlusion and vestibular nerve resection was excluded from the analyses.

TSCO. However, one patient whose main complaint was severe dizziness rather than vertigo had no benefit after surgery. We also counted the postoperative vertigo control of the two groups regardless of the postoperative time. The comparison of vertigo control between the two groups was unchanged (all $P>0.05$; Table 3).

Ten patients in the TSCO group completed four questionnaires via telephone during follow-up. Based on the 
TABLE 2 | Vertigo control after surgery in patients who performed surgery more than 24 months ago.

\begin{tabular}{|c|c|c|c|c|}
\hline & Total $(N=14)$ & $\operatorname{TSCO}\left(N=4^{\star}\right)$ & VNS $(N=10)$ & $P$-value \\
\hline Number of vertigo episodes in 24 months after surgery & $0.07 \pm 0.28$ & $0.00 \pm 0.00$ & $0.10 \pm 0.32$ & 0.839 \\
\hline \multicolumn{5}{|l|}{ Class } \\
\hline A & $13(93 \%)$ & $4(100 \%)$ & $9(90 \%)$ & 0.512 \\
\hline$B$ & $1(7 \%)$ & $0(0 \%)$ & $1(10 \%)$ & \\
\hline
\end{tabular}

*Four of 10 patients in the triple semicircular canal occlusion group were operated on more than 24 months ago.
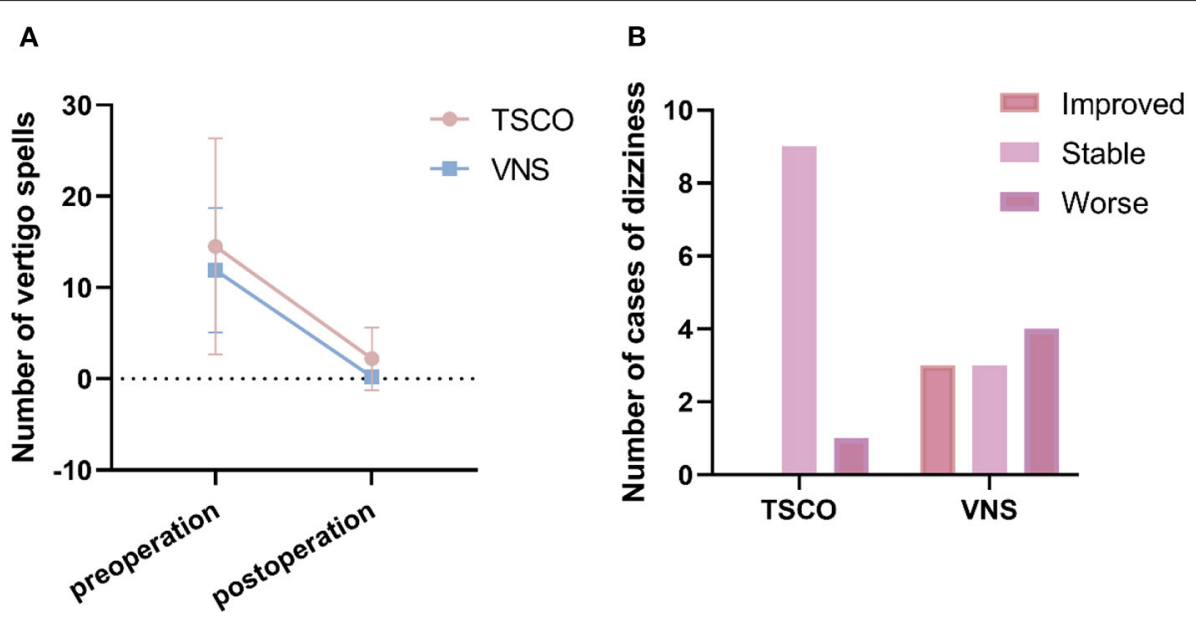

FIGURE 2 | Incidence of postoperative vertigo and paroxysmal dizziness. (A) Vertigo spells were controlled by both triple semicircular canal occlusion (TSCO) and vestibular nerve resection (VNS), and there was no significant difference in incidence between the two groups. (B) Bar chart comparing the numbers of postoperative cases of paroxysmal dizziness between the two groups.

DHI results, we evaluated the patients' quality of life physically, emotionally, and functionally; a large group difference was found in all three domains $(P=0.022,0.025$, and 0.013 , respectively). The scores were significantly lower in patients who underwent TSCO more than 24 months ago compared to those operated on more recently. However, no significant differences were found in the ABC, THI, or VAS scores (all $P>0.05$; Table 4).

\section{Postoperative Paroxysmal Dizziness and Unsteadiness}

Postoperative problems requiring attention include paroxysmal dizziness (sensation of disturbed or impaired spatial orientation without a distorted sense of motion), unsteadiness (postural instability when sitting, standing, or walking (19)), and vertigo recurrence. As one of the symptoms of vestibular function disorder dizziness may coexist with vertigo in Menière's disease. In terms of paroxysmal dizziness, different patients have different conditions. Using the number of patients with postoperative paroxysmal dizziness to analyze was improper for losing the individual characteristics of patients. All patients were encouraged to exercise and no specific vestibular physical therapy was performed. After surgery we scored the symptoms of our patients as follows: 1, improved: dizziness had occurred before but disappeared after the operation; 2, stable: dizziness existed before and after the operation (or asymptomatic patients); or
3, worse: no dizziness previously, but dizziness onset after the operation. The data indicated that TSCO was significantly better than VNS in reducing dizziness $(P=0.020$; Table 5; Figure 2B). unsteadiness, which is a symptom of vestibular dysfunction, only occurred after VNS $(P=0.025)$.

\section{Special Case}

One patient in our study underwent both TSCO and VNS. He underwent ESS for recurrent vertigo in 2002 but it recurred. Therefore, in 2005, he underwent VNS and vertigo control was achieved. However, dizziness and unsteadiness occurred after this surgery and lasted for 10 years. In 2016, his vertigo recurred with attacks lasting $2 \mathrm{~h}$. After undergoing TSCO in 2017, his vertigo and dizziness disappeared completely, but functional compensation for the unsteadiness was difficult to achieve.

\section{DISCUSSION}

In this retrospective trial, we found no significant difference between TSCO and VNS in terms of the ability to control vertigo. At 24 months after surgery, all patients in the TSCO group achieved class A vertigo control, whereas there were nine cases of class A vertigo, and 1 of class B, in the VNS group. The results confirm the efficacy of the two surgical methods for controlling vertigo symptoms. However, other 
TABLE 3 | Vertigo control after surgery in all follow-up patients.

\begin{tabular}{lccc}
\hline & Total $(\boldsymbol{N}=\mathbf{2 0})$ & TSCO $(\boldsymbol{N}=\mathbf{1 0})$ & VNS (N=10) \\
\hline Number of vertigo episodes & $1.15 \pm 2.60$ & $2.20 \pm 3.43$ & $0.10 \pm 0.32$ \\
Class & $15(75 \%)$ & $6(60 \%)$ & $9(90 \%)$ \\
A & $4(20 \%)$ & $3(30 \%)$ & $1(10 \%)$ \\
B & $1(5 \%)$ & $1(10 \%)$ & 0.273 \\
D & & $(0 \%)$ & \\
\hline
\end{tabular}

TABLE 4 | Dizziness handicap inventory, activities-specific balance confidence scale, tinnitus handicap inventory, and visual analog scale scores of the triple semicircular canal occlusion patients.

\begin{tabular}{|c|c|c|c|c|}
\hline & Total $(N=10)$ & $\begin{array}{l}\text { Operated on within } \\
\text { the last } 24 \text { months } \\
\qquad(N=6)\end{array}$ & Operated on more than 24 months ago $(N=4)$ & $P$-value \\
\hline DHI-E & $11.33 \pm 12.73$ & $19.20 \pm 12.13$ & $1.50 \pm 1.92$ & 0.025 \\
\hline $\mathrm{DHI}-\mathrm{F}$ & $14.67 \pm 17.75$ & $26.00 \pm 16.37$ & $0.50 \pm 1.00$ & 0.013 \\
\hline VAS (1-10) & $3.50 \pm 3.32$ & $5.20 \pm 3.56$ & $1.38 \pm 1.25$ & 0.082 \\
\hline
\end{tabular}

vestibular symptoms including dizziness and unsteadiness were less common among TSCO than VNS patients. It is plausible that the vestibular nerve on the healthy side cannot completely compensate for the balance disorders caused by VNS on the affected side. Our study also showed that the DHI scores of patients more than 24 months after TSCO were significantly lower than those within 24 months, reflecting a change in patient emotional, physical, and functional quality of life. Vestibular function recovery in TSCO patients takes time.

Fan et al. (24) reported a $100 \%$ success rate for vertigo control after TSCO, with $81.6 \%$ and $18.4 \%$ of patients achieving complete and partial control, respectively, after 2 years (25). Setty et al. (26) reported that vertigo was controlled in $97.7 \%$ of Menière's disease patients after VNS. Similarly high rates of control of vertigo have also been reported in other studies. However, researchers often ignore postoperative symptoms surgeons can only choose the most effective surgical method by studying the relationships among vertigo and dizziness, unsteadiness, and vestibular disorder.

Surgical ablative therapies are considered an option for patients with active Menière's disease who have failed less definitive treatments and have unusable hearing (3). For nearly a century the VNS operation has been used to treat intractable Menière's disease (since the 1920s (11)). VNS controls vertigo by cutting the vestibular nerve, which sacrifices vestibular function on the lesioned side (11). This could explain why unsteadiness, dizziness attacks, and residual dizziness occur more often in VNS than TSCO patients, with the former group correspondingly showing larger changes in postoperative quality of life. By contrast, TSCO only blocks endolymph flow in three semicircular canals, which decreases vestibular function only on the surgical side (13). Overall, TSCO is equally effective as VNS for vertigo control, but is associated with a lower rate of dizziness.

A major concern with TSCO is postoperative HL. Stultiens et al. (27) assessed the postoperative hearing of patients with a semicircular canal plug and found a difference from the preoperative hearing level of $<10 \mathrm{~dB} \mathrm{HL}$ at the 6-month followup. Their study included stage 1-2 patients. In 2015 Zhang et al. (25) reported a rate of hearing preservation of $69.4 \%$ for TSCO. In the present study, the patients who underwent TSCO were mostly in stage 4 , so it was difficult to determine whether TSCO was helpful for postoperative hearing recovery and preservation.

VNS is an intracranial operation with potential risks for cerebrospinal fluid leaks and facial nerve injury (28). In our patients none of these occurred. Risk for facial nerve injury can be decreased by a retrosigmoid approach (28). Watertight sutures of meninges, use of mannitol, and raising the head to 30 degrees postoperatively will minimize cerebrospinal fluid leaks.

There are some other surgical approaches available for refractory Menière's disease. According to a survey by the American Academy of Otolaryngology-Head and Neck Surgery Foundation, ESS was the initial surgical intervention for the treatment of Menière's disease in $50 \%$ of the respondents (7). Portmann et al. (29) first described ESS in 1927. Saliba et al. (30) reported that the vertigo control rate was $37.5 \%$ 4 months after ESS. The main advantage of this surgery is that the hearing is preserved, however, no significant difference in symptom improvement was found between ESS and placebo groups in a study by Bretlau (31), although no reported complications or side effects were found either. Despite this controversy over its effectiveness, ESS is still widely used (32). 
TABLE 5 | Comparison of postoperative symptoms between the two groups.

\begin{tabular}{|c|c|c|c|c|}
\hline & Total $(N=20)$ & $\operatorname{TSCO}(N=10)$ & VNS $(N=10)$ & $P$-value \\
\hline \multicolumn{5}{|l|}{ Dizziness } \\
\hline Improved (n/\%) & $3(15 \%)$ & $0(0 \%)$ & $3(30 \%)$ & 0.020 \\
\hline Stable (n/\%) & $12(60 \%)$ & $9(90 \%)$ & $3(30 \%)$ & \\
\hline Worse (n/\%) & $5(25 \%)$ & $1(10 \%)$ & $4(40 \%)$ & \\
\hline Unsteadiness ( $n / \%)$ & $4(18 \%)$ & $0(0 \%)$ & $4(36 \%)$ & 0.025 \\
\hline
\end{tabular}

Parnes et al. (21) found that blockage of a semicircular canal did not influent the function of the other canal receptors. TSCO, which has the same principle, may preserve some vestibular and hearing function on the side of surgery (13). The result of v-HIT showed a decrease of function of semicircular canals, which increased the threshold of vertigo attack (33). Animal models have indicated that endolymphatic movement caused by excessive accumulation of endolymph will decrease or disappear after semicircular canal obstruction theoretically (13). TSCO exerts its effects as follows. Occlusions partially prevent the endolymph from stimulating the semicircular canals, and displacement of the crista ampullaris (which contributes to rotational vertigo). Also, postoperative disequilibrium is quickly compensated for (34). Our study provides guidance on the surgical method for Menière's disease patients, by demonstrating that TSCO is an alternative choice for vertigo control.

\section{LIMITATIONS}

There were several limitations to our study. First, we estimated the effects of surgery via pre- and post-operative comparison, which limited the sample size given the lack of postoperative auditory and vestibular evaluation results in some cases. This bias may have affected the accuracy of the analysis. Also, we may have underestimated the rate of postoperative vestibular symptoms given the small sample size, and the long follow-up time might have led to recall bias. Finally, postoperative data for pure-tone audiometry, for example, were incomplete, which limited our audiological evaluation. However, these limitations did not prevent us from describing changes of vestibular symptoms after surgery. Large-scale research using the same pre- and post-operative tests applied in the present study is required.

\section{CONCLUSION}

The difficulty of treating refractory Menière's disease is well recognized, so selecting the optimal surgical method remains

\section{REFERENCES}

1. Lopez-Escamez JA, Carey J, Chung WH, Goebel JA, Magnusson M, Mandala M, et al. Diagnostic criteria for Meniere's disease. J Vestib Res. (2015) 25:17. doi: 10.3233/VES-150549

2. Watanabe Y, Mizukoshi K, Shojaku H, Watanabe I, Hinoki M, Kitahara M. Epidemiological and clinical characteristics of Meniere's disease in challenging. Our study shows that TSCO controls vertigo in most Menière's disease patients, and also has the advantage of lower rates of postoperative paroxysmal dizziness and unsteadiness than VNS. Thus, TSCO can be considered as an effective surgery for refractory Menière's disease.

\section{DATA AVAILABILITY STATEMENT}

The raw data supporting the conclusions of this article will be made available by the authors, without undue reservation.

\section{ETHICS STATEMENT}

The studies involving human participants were reviewed and approved by Shanghai Jiao Tong University Affiliated Sixth People's Hospital (2020 KY 004). Written informed consent for participation was not required for this study in accordance with the national legislation and the institutional requirements.

\section{AUTHOR CONTRIBUTIONS}

DY and ZC: designed and coordinated the study. YJ, QY, $Z L$, and MX: analyzed the data and wrote the manuscript. DY, ZC, YW, HS, and SY: performed the surgery. ZC and DY: attests that all listed authors meet authorship criteria and that no others meeting the criteria have been omitted. All authors contributed to the article and approved the submitted version.

\section{FUNDING}

This work was supported by Shanghai Municipal Education Commission-Gaofeng Clinical Medicine Grant (20191921) and National Key Research and Development Project (2019YFC0119900). 
a systematic review and meta-analysis. Otolaryngol Head Neck Surg. (2019) 160:232-8. doi: 10.1177/0194599818803612

5. Vlastarakos PV, Iacovou E, Nikolopoulos TP. Is gentamycin delivery via sustained-release vehicles a safe and effective treatment for refractory Meniere's disease? A critical analysis of published interventional studies. Eur Arch Otorhinolaryngol. (2017) 274:1309-15. doi: 10.1007/s00405-016-4294-9

6. da Costa SS, de Sousa LC, Piza MR. Meniere's disease: overview, epidemiology, and natural history. Otolaryngol Clin North Am. (2002) 35:45595. doi: 10.1016/S0030-6665(02)00028-2

7. Kim HH, Wiet RJ, Battista RA. Trends in the diagnosis and the management of Meniere's disease: results of a survey. Otolaryngol Head Neck Surg. (2005) 132:722-6. doi: 10.1016/j.otohns.2005.01.007

8. Claes J, Van de Heyning PH. A review of medical treatment for Meniere's disease. Acta Otolaryngol Suppl. (2000) 544:349. doi: 10.1080/000164800750044461

9. Santos PM, Hall RA, Snyder JM, Hughes LF, Dobie RA. Diuretic and diet effect on Meniere's disease evaluated by the 1985 Committee on Hearing and Equilibrium guidelines. Otolaryngol Head Neck Surg. (1993) 109:6809. doi: $10.1177 / 019459989310900408$

10. Pearson BW, Brackmann DE. Committee on hearing and equilibrium guidelines for reporting treatment results in Meniere's disease. Otolaryngol Head Neck Surg. (1985) 93:579-81. doi: 10.1177/019459988509300501

11. Silverstein H, Jackson LE. Vestibular nerve section. Otolaryngol Clin North Am. (2002) 35:655-73. doi: 10.1016/S0030-6665(02)00024-5

12. Parnes LS, Agrawal SK, Atlas J. Diagnosis and management of benign paroxysmal positional vertigo (BPPV). CMAJ. (2003) 169:681-93

13. Yin $\mathrm{S}, \mathrm{Yu} \mathrm{D}, \mathrm{Li} \mathrm{M}$, Wang J. Triple semicircular canal occlusion in guinea pigs with endolymphatic hydrops. Otol Neurotol. (2006) 27:7885. doi: 10.1097/01.mao.0000170535.42023.96

14. Yin S, Chen Z, Yu D, Wu Y, Shi H, Zhou H, et al. Triple semicircular canal occlusion for the treatment of Meniere's disease. Acta Otolaryngol. (2008) 128:739-43. doi: 10.1080/00016480701730000

15. Zhang D, Fan Z, Han Y, Lv Y, Li Y, Wang H. Triple semicircular canal plugging: a novel modality for the treatment of intractable Meniere's disease. Acta Otolaryngol. (2016) 136:1230-5. doi: 10.1080/00016489.2016.1206966

16. Ahmadzai N, Cheng W, Kilty S, Esmaeilisaraji L, Wolfe D, Bonaparte J, et al. Pharmacologic and surgical therapies for patients with Meniere's disease: a systematic review and network meta-analysis. PLoS ONE. (2020) 15:e237523. doi: 10.1371/journal.pone. 0237523

17. Abdala C, Visser-Dumont L. Distortion product otoacoustic emissions: a tool for hearing assessment and scientific study. Volta Rev. (2001) 103:281-302.

18. Jabłonka A, Pośpiech L, Orendorz-Fraczkowska K. Evaluation of glycerol test in Meniere's disease with pure tone audiometry and distortion product otoacoustic emission. Otolaryngologia polsk $a=$ The Polish otolaryngology. (2003) 57:731-737.

19. Bisdorff A, Von Brevern M, Lempert T, Newman-Toker DE. Classification of vestibular symptoms: towards an international classification of vestibular disorders. J Vestib Res. (2009) 19:1-13. doi: 10.3233/VES-20090343

20. Kim SK, Kim JH, Jeon SS, Hong SM. Relationship between sleep quality and dizziness. PLoS ONE. (2018) 13:e0192705. doi: 10.1371/journal.pone.0192705

21. Parnes LS, McClure JA. Posterior semicircular canal occlusion for intractable benign paroxysmal positional vertigo. Ann Otol Rhinol Laryngol. (1990) 99:330-4. doi: 10.1177/0003489490099 00502

22. Silverstein H, Nichols ML, Rosenberg S, Hoffer M, Norrell H. Combined retrolabyrinthine-retrosigmoid approach for improved exposure of the posterior fossa without cerebellar retraction. Skull Base Surg. (1995) 5:17780. doi: 10.1055/s-2008-1058932
23. Pareschi R, Destito D, Falco Raucci A, Righini S, Colombo S. Posterior fossa vestibular neurotomy as primary surgical treatment of Meniere's disease: a re-evaluation. J Laryngol Otol. (2002) 116:593-6. doi: 10.1258/00222150260171560

24. Fan ZM, Zhang DG, Han YC, Wang HB. Short-term efficacy of semicircular canal occlusion in the treatment of intractable Meniere's disease. Zhonghua Er Bi Yan Hou Tou Jing Wai Ke Za Zhi. (2012) 47:6779 doi: 10.1055/s-0032-1314349

25. Zhang D, Fan Z, Han Y, Li Y, Wang H. Long-term efficacy of triple semicircular canal occlusion in the treatment of intractable Meniere's disease. Zhonghua Er Bi Yan Hou Tou Jing Wai Ke Za Zhi. (2015) 50:733-7.

26. Setty P, Babu S, LaRouere MJ, Pieper DR. Fully endoscopic retrosigmoid vestibular nerve section for refractory Meniere disease. J Neurol Surg B Skull Base. (2016) 77:341-9. doi: 10.1055/s-0035-1570348

27. Stultiens JJA, Guinand N, Van Rompaey V, Perez Fornos A, Kunst HPM, Kingma $\mathrm{H}$, et al. The resilience of the inner ear-vestibular and audiometric impact of transmastoid semicircular canal plugging. J Neurol. (2021) 10:10. doi: 10.1007/s00415-021-10693-5

28. Alarcon AV, Hidalgo LO, Arevalo RJ, Diaz MP. Labyrinthectomy and vestibular neurectomy for intractable vertiginous symptoms. Int Arch Otorhinolaryngol. (2017) 21:184-90. doi: 10.1055/s-0037-1599242

29. Portmann G. Surgical treatment of vertigo by opening of the saccus endolymphaticus. Arch Otolaryngol, (1969) 89:809-15 doi: 10.1001/archotol.1969.00770020811005

30. Saliba I, Gabra N, Alzahrani M, Berbiche D. Endolymphatic duct blockage: a randomized controlled trial of a novel surgical technique for Meniere's disease treatment. Otolaryngol Head Neck Surg. (2015) 152:1229. doi: $10.1177 / 0194599814555840$

31. Cooper MW, Kaylie DM. Is Endolymphatic Sac Surgery Beneficial For Meniere's Disease? Laryngoscope. (2020) 130:2738-9. doi: 10.1002/lary.28647

32. Sood AJ, Lambert PR, Nguyen SA, Meyer TA. Endolymphatic sac surgery for Meniere's disease: a systematic review and meta-analysis. Otol Neurotol. (2014) 35:1033-45. doi: 10.1097/MAO.0000000000000324

33. Zhang D, Lv Y, Han Y, Sun G, Li Y, Li X, et al. Revision surgery after triple semicircular canal plugging and morphologic changes of vestibular organ. Sci Rep. (2019) 9:19397. doi: 10.1038/s41598-019-55810-7

34. Liu F, Huang W, Chen K. Clinical long-term effects of surgical treatment for intractable Meniere's disease: a more than 13-year follow-up after pressure treatment and further surgical treatment for intractable vertigo. Acta Otolaryngol. (2019) 139:1053-7. doi: 10.1080/00016489.2019.1667532

Conflict of Interest: The authors declare that the research was conducted in the absence of any commercial or financial relationships that could be construed as a potential conflict of interest.

Publisher's Note: All claims expressed in this article are solely those of the authors and do not necessarily represent those of their affiliated organizations, or those of the publisher, the editors and the reviewers. Any product that may be evaluated in this article, or claim that may be made by its manufacturer, is not guaranteed or endorsed by the publisher.

Copyright $\odot 2022$ Jiang, Xu, Yao, $L i, W u$, Chen, Yu, Shi and Yin. This is an openaccess article distributed under the terms of the Creative Commons Attribution License (CC BY). The use, distribution or reproduction in other forums is permitted, provided the original author(s) and the copyright owner(s) are credited and that the original publication in this journal is cited, in accordance with accepted academic practice. No use, distribution or reproduction is permitted which does not comply with these terms. 